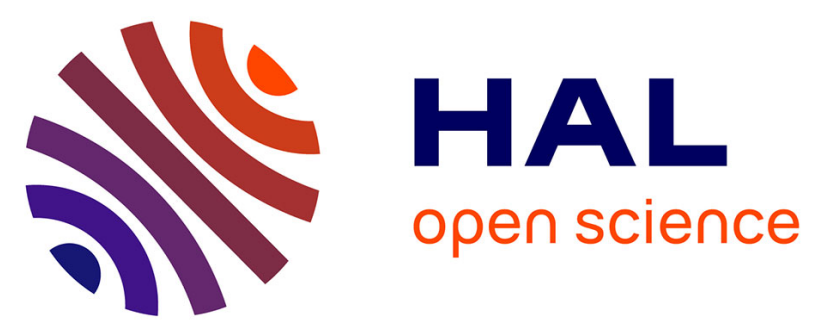

\title{
Seasonal variability of degrees of freedom and its effect over time series and spatial patterns of atmospheric gases from satellite: application to carbonyl sulfide (OCS)
}

Carmine Serio, Guido Masiello, Pietro Mastro, Sauveur Belviso, Marine

Remaud

\section{To cite this version:}

Carmine Serio, Guido Masiello, Pietro Mastro, Sauveur Belviso, Marine Remaud. Seasonal variability of degrees of freedom and its effect over time series and spatial patterns of atmospheric gases from satellite: application to carbonyl sulfide (OCS). Proceedings of SPIE, the International Society for Optical Engineering, 2021, 11859, Remote Sensing of Clouds and the Atmosphere XXVI, pp.1185904. 10.1117/12.2599761. hal-03351801

\author{
HAL Id: hal-03351801 \\ https://hal.science/hal-03351801
}

Submitted on 11 Oct 2021

HAL is a multi-disciplinary open access archive for the deposit and dissemination of scientific research documents, whether they are published or not. The documents may come from teaching and research institutions in France or abroad, or from public or private research centers.
L'archive ouverte pluridisciplinaire HAL, est destinée au dépôt et à la diffusion de documents scientifiques de niveau recherche, publiés ou non, émanant des établissements d'enseignement et de recherche français ou étrangers, des laboratoires publics ou privés. 


\title{
Seasonal variability of degrees of freedom and its effect over time series and spatial patterns of atmospheric gases from satellite: application to carbonyl sulfide (OCS)
}

\author{
Carmine Serio $^{\mathrm{a}}$, Guido Masiello ${ }^{\mathrm{a}}$, Pietro Mastro ${ }^{\mathrm{a}}$, Sauveur Belviso ${ }^{\mathrm{b}}$, and Marine Remaud ${ }^{\mathrm{b}}$ \\ ${ }^{a}$ Scuola di Ingegneria, Università degli Studi della Basilicata, via Ateneo Lucano, 10, 85100 \\ Potenza, Italy \\ ${ }^{b}$ Laboratoire des Sciences du Climat et de l'Environnement, CE Saclay, Orme des Merisiers, \\ 91191 Gif-sur-Yvette, France
}

\begin{abstract}
Degrees of freedom or d.o.f. of satellite-based retrievals characterize their independence from the constraints assumed in the inversion process. In the context of Optimal Estimation (OE), the condition is expressed in terms of the background state, which, in a Bayesian meaning, is our best prior knowledge about the parameters we want to estimate. In effect, even if the background is static, it could add artifacts to the retrievals, which modify the seasonal cycle or the spatial patterns of 2-D fields. The issue has been addressed with an analytical treatment based on the OE theory. We derive formulas, which allow us to assess the modulation introduced by d.o.f. variability. The methodology will be exemplified with the help of observations from the Infrared Atmospheric Sounder Interferometer (IASI) onboard the European MetOp satellites. Both time series and 2-D fields of observations will be considered. The analysis will focus mainly on OCS (carbonyl sulfide) variability in the atmosphere, a new clue to how much carbon plants take up, hence of primary interest to the carbon cycle and the climate. However, our methodology can be applied to any gas or retrieved parameter. For the OCS, we have found that d.o.f. variability is of no concern in the tropics. Still, it becomes crucial at Mid-latitudes where the seasonal cycle can add spurious variability to temporal and spatial patterns.
\end{abstract}

Keywords: Satellite, atmospheric gases, carbon cycle, carbonyl sulfide, infrared, sounders, forward modeling, inverse problems, optimal estimation

\section{INTRODUCTION}

Nowadays, satellite level 2 algorithms for the estimations of gas species and thermodynamic parameters make widespread use of Optima Estimation or $\mathrm{OE},{ }^{1}$ a Bayesian approach that constrains the solution through the background state, which is our best prior knowledge about the parameters. In this context, the concept of degrees of freedom or d.o.f. of satellite-based retrievals is used, which measures the independence of the background from the final or level 2 product we get through the inversion process. Usually, the background constraint is a static feature of the inversion process, and therefore, in principle, it should not affect, e.g., the seasonality of the retrieved quantities, although a too much strong constraint can attenuate the dynamic of the estimated products, hence the amplitude of the seasonal cycle. However, we will show that there are other factors which do not depend on the background but on the atmospheric state, which can even change the phase of the seasonal cycle. Understanding and assessing the role of the background is an important issue, e.g., when we need to analyze the seasonal cycle of a given atmospheric gas, such as $\mathrm{CO}_{2}$ or other greenhouse gases. Then, we can be confident that the variability we see in the satellite products is a natural effect coming from the observations and not the background. In effect, even if the background is static, it could add artifacts to the retrievals, which modify the seasonal cycle or the spatial patterns of 2-D fields. To avoid any spurious behaviors, we simultaneously need the background to be static and d.o.f. to have precisely the same value for every retrieval. In case the d.o.f. changes over time and spatial locations, the retrievals can be modulated by the d.o.f. variability to the

(Send correspondence to C.S.)

carmine.serio@unibas.it 
extent that what we see in the time series or spatial fields is nothing but the d.o.f. behavior. The issue has been addressed with an analytical treatment based on the OE theory. We derive formulas, which allow us to assess the modulation introduced by d.o.f. variability. The Infrared Atmospheric Sounder Interferometer (IASI) ${ }^{2}$ on board the European MetOp satellites have been used to analyze the problem quantitatively. Both time series and 2-D fields of observations will be considered. The analysis is extended to tropical and Mid-Lat regions to exemplify the effect of seasonal variability of d.o.f.. Although the methodology we will discuss is quite general, the study will focus mainly on retrieving carbonyl sulfide or OCS, which is of primary interest to the carbon cycle and the climate. In effect, OCS is receiving increasing attention (e.g., see Refs. 3-5) because (1) the fluxes of $\mathrm{CO}_{2}$ and OCS into leaves follow similar pathways through plant stomata and (2) the enzyme carbonic anhydrase catalyses both the hydration of $\mathrm{CO}_{2}$ and the hydrolysis of OCS. However, unlike $\mathrm{CO}_{2}$ hydration, the hydrolysis of OCS is an irreversible reaction, which means that the captured OCS can no longer be released back to the atmosphere. Thus, the OCS absorption by plants is a potentially helpful proxy of $\mathrm{CO}_{2}$ absorption, and therefore it is directly related to gross primary production (GPP). Attempts to retrieve OCS from the satellite are relatively recent, e.g., see Refs. 6-11, although the quality and reliability of the retrieval have not yet been assessed accurately. This gap will be filled in the present paper. The study is organized as follows. Section 2 will deal with data and methods, whereas results will be shown in section 3. Finally, conclusions will be drawn in section 4 .

\section{DATA AND METHODOLOGY}

\subsection{Data: in situ observations}

Representative OCS flask monthly data (e.g., see Ref. 12) were collected at the station of Mauna Loa (short name MLO, $19.5362 \mathrm{~N}, 155.5763 \mathrm{~W}, 3397 \mathrm{~m}$ asl). MLO observatory (see Fig. 1) in Hawaii is part of NOAA Earth System Research Laboratory (ESRL) and Global Monitoring Division (GMD). The data considered in this analysis refers to the period 2014-2019.

In addition to the tropical OCS data, we have also collected observations from a mid-lat station located at Gif-sur-Yvette, France (short name GIF, 48.701N, 2.1475 W, $165 \mathrm{~m}$ asl, $7 \mathrm{~m} \mathrm{agl}$ ). At GIF station (see Fig. 1), hourly measurements have been performed for six and a half years (starting from 2014) using online gas chromatography with Pulsed Flame Photometry detection (see Ref. 13). More detailed information about the calibration method can be found in Ref. 13. It is worth noting that data accuracy was systematically assessed using a NOAA/ESRL certified standard for COS, proven to exhibit no long-term drift, as target gas (e.g., see Ref. 14). Hence, we can state that in that sense, GIF data are calibrated on the NOAA-2004 scale.
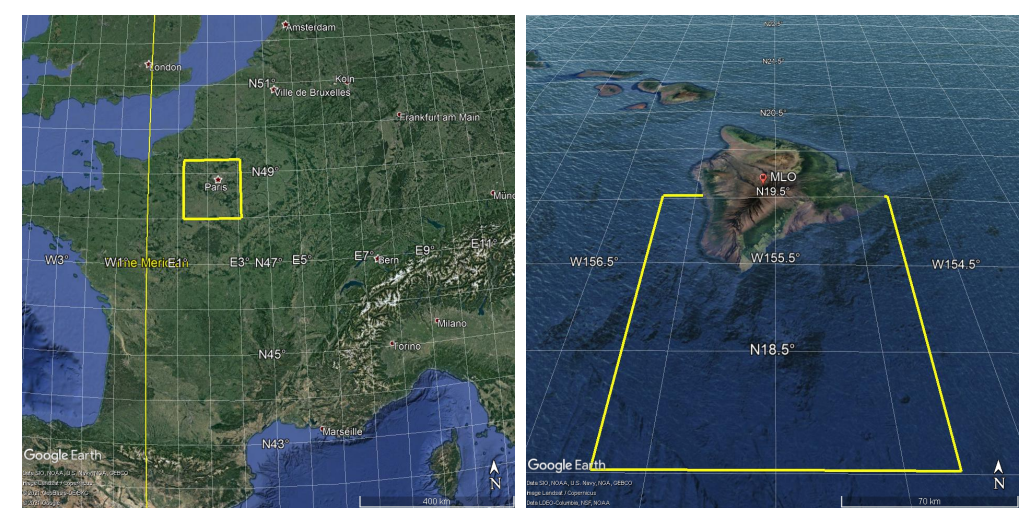

Figure 1. Left: GIF target area; right: Hawaii target area. The yellow box shows the site for which IASI soundings have been acquired. In both figures, the red tag indicates the location of the OCS monitoring stations.

\subsection{Data: IASI observtaions}

For comparison, IASI OCS observations were obtained for the GIF and Hawaii target areas shown in the map of 1. The IASI soundings cover 2014-2019. Only clear sky soundings have been considered. To check the impact of spatial degrees of freedom variability, we have also considered IASI soundings for the European and 
Mediterranean (Euro-Med) area shown in Fig. 2. The data acquired for this area refers to February 2020. We stress that all the IASI data used in this paper consist of clear sky soundings. Spectra were filtered for the clear sky by using the native cloud mask of IASI Level $1 \mathrm{C}$ data. This cloud mask is released by EUMETSAT and is based on the Advanced Very High-Resolution Radiometer or AVHRR imagery. A further cloud detection scheme was also used (e.g., see Refs. 15-17) and applied sequentially to the previous one to improve the quality of the final results.

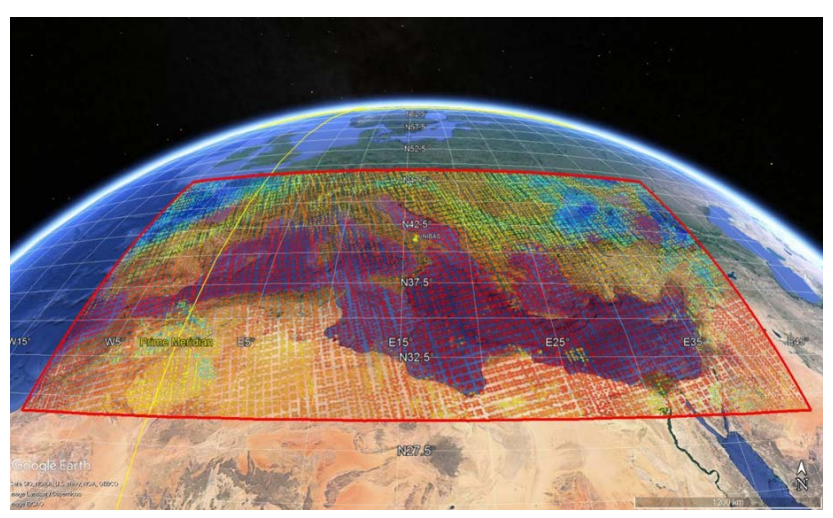

Figure 2. Euro-Med target area (red box) used to assess the effect of d.o.f. variability on spatial patterns.

\subsection{Basic Methodology}

OCS retrievals from IASI have been obtained through an inversion algorithm, combining a fully analytical, monochromatic radiative transfer model and an Optimal Estimation (OE) module. ${ }^{1}$ The radiative transfer model is called IASI ${ }^{9,18}$ and is a fast model, which uses a pre-computed look-up table for the optical depth calculation. The look-up table is derived from LBLRTM (Line-by-Line Radiative Transfer Model, e.g., see Refs 19,20), using the line compilation aer 3.2 (see e.g., http://rtweb.aer.com/line_param_frame.html). The forward module computes the radiance after discretizing the radiative transfer equation on a fixed grid made up by $N_{L}=60$ pressure layers, which spans the atmosphere from 1050 to $0.005 \mathrm{hPa}$. The forward model yields spectral radiances and analytical Jacobians concerning surface temperature, emissivity, and any atmospheric parameter, including the volume mixing ratio of the gases mentioned above. In its latest version, ${ }^{9} \sigma$-IASI also considers the sun spectral radiation, which may add an essential contribution in the spectral interval 2000 to $2240 \mathrm{~cm}^{-1}$, which contains most information for retrieving OCS. The inversion scheme, called $\delta$-IASI,${ }^{21}$ implements an iterative algorithm for the optimal estimation of the thermodynamic state of the atmosphere and its composition. The model $\delta$ IASI performs the mathematical inversion of the whole IASI radiance spectrum (8461 channels) to simultaneously retrieve the state vectors $\left(\varepsilon, T_{s}, T, Q, O, H D O, \mathrm{CO}_{2}, \mathrm{~N}_{2} \mathrm{O}, \mathrm{CO}, \mathrm{CH}_{4}, \mathrm{SO}_{2}, \mathrm{HNO}_{3}, \mathrm{NH}_{3}, O C S, \mathrm{CF}_{4}\right)$. A critical aspect of the retrieval scheme, which is relevant to the estimation of trace gases, is the use of background and first guess state vectors in the linear region of the radiative transfer equation. Towards this objective, we use ECMWF (European Centre for Medium range Weather Forecasts) analysis for $(T, Q, O)$ collocated in space and time with the IASI soundings (see, e.g., Refs. 9, 10 for details). ECMWF $(T, Q, O)$ analysis provides a strong constraint in the inverse scheme, and we think that the corresponding profiles are reliable and allow us to relax the conditioning for the trace gases. In effect, minor and trace gas profiles are usually adjusted starting from a climatology as a first guess. The retrieved profiles reach their final values in one iteration. For OCS, the background profile for the corresponding part of the state vector is set equal to the reference profile (see Fig. 3).

In addition to the background state, we also need to specify a proper background covariance matrix. We consider a Markov-type matrix, and we refer the interested reader to Ref. 10 for further details about this matrix. It should be stressed that the covariance matrix is set so that it applies a loose constraint. Because of the possible d.o.f. modulation over the retrieval, our objective is to set up a covariance matrix so that the final OCS product is as much as possible independent of the a prior profile. 

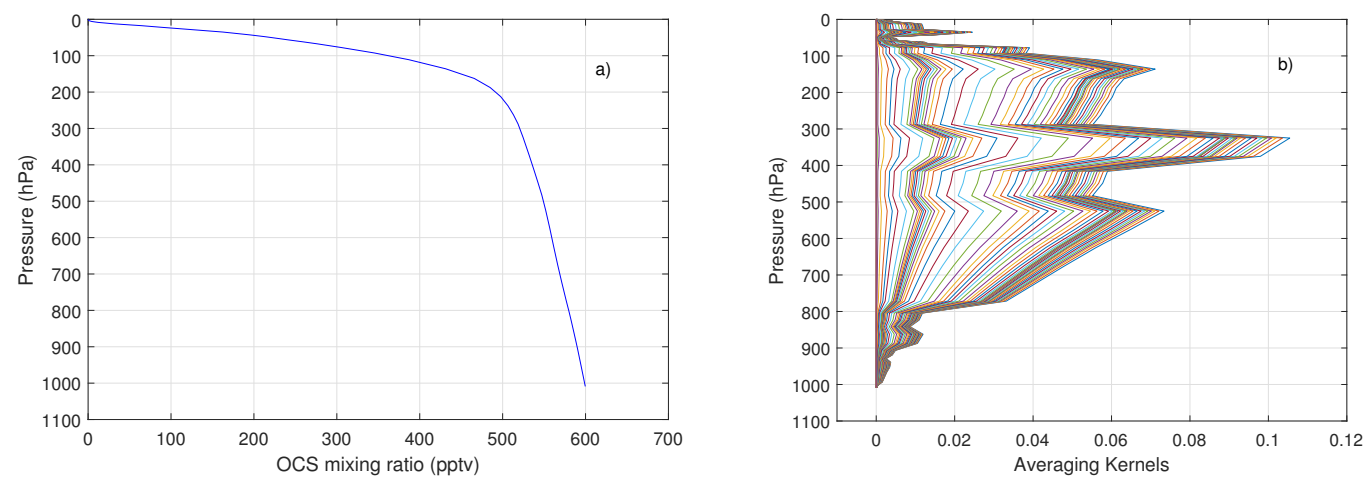

Figure 3. a)- OCS background profile used for the retrieval from IASI data. b)- Example of Averaging Kernels for OCS.

\subsubsection{OCS retrieval details: the role of the data and the background}

This section aims at deriving a set of equations that can give us a quantitative measure of the contribution of the background and data to the column amount retrieval of the prescribed gas species or atmospheric parameter. An essential aspect of the derivation presented in this section is how to formalize, with appropriate equations, the time-space evolution of the contribution coming from the a prior constraint. In effect, one could argue that based on Fig. 3, and considering that we have fixed the background, its contribution needs to be static. However, we will show that this is not the case because the background contribution to the retrieval also depends on the given state of the atmosphere, which can change with season and space.

To begin with, we note that in our scheme, an optimal estimate of the column amount of OCS, $\hat{X}_{O C S}$ is obtained from a retrieved vector-valued profile $\hat{\mathbf{q}}$ OCS of size $N_{L}$ (i.e., the number of layers in our forward model)

$$
\hat{\mathbf{X}}_{O C S}=\mathbf{h}^{t} \hat{\mathbf{q}}_{\text {oCs }} ; \quad \operatorname{var}\left(\hat{X}_{O C S}\right)=\mathbf{h}^{t} \mathbf{S}_{\hat{\mathbf{q}}} \mathbf{h}
$$

where $\mathbf{S}_{\hat{\mathbf{q}}}$ is the retrieval covariance matrix, $\mathbf{h}$ the averaging operator (e.g., see Ref. 10) and var $(\cdot)$ stands for variance. Because our methodology can be applied to any parameter, hereafter, the subscript OCS will be omitted for the sake of generality. Having said that, the column amount $\hat{\mathbf{X}}_{O C S}$ is corrected for the effect of moist air, as shown in Ref. 10. The averaging operator, $\mathbf{h}$ is defined according to the quadrature formula we use to compute the column amount from the mixing ratio profile, that is

$$
\hat{X}=-\frac{1}{p_{1}-p_{N_{L}+1}} \int_{p_{1}}^{p_{N_{L}+1}} \hat{q} d p=\frac{1}{p_{1}-p_{N_{L}+1}} \sum_{i=1}^{N_{L}} \hat{q}_{i}\left(p_{i+1}-p_{i}\right)
$$

with $p_{i}$ the pressure of the level $i, i=1, \ldots, N_{L}$. We define

$$
h_{i}=\frac{p_{i}-p_{i+1}}{p_{1}-p_{N_{L}}+1}
$$

(with $p_{1}$ the surface pressure), and Eq. (2) becomes,

$$
\hat{X}=\sum_{i=1}^{N_{L}} h_{i} \hat{q}_{i}=\mathbf{h}^{t} \hat{\mathbf{q}}
$$

It should be also noted that because of its definition, we have

$$
\sum_{i=1}^{N_{L}} h_{i}=1
$$


which means that the weights are appropriately normalized.

Figure 3b shows a typical Averaging Kernels (AK) for the retrieved OCS profile, from which we derive a value $\approx 1$ for the degrees of freedom, computed as the trace of AK. However, we are mostly interested in the retrieval of the column amount, and, therefore, in the Averaging Kernel for $\hat{X}$. Towards this objective, if $\mathbf{A}$ is the averaging kernels matrix for OCS profile, that for the column amount is $\mathbf{h}^{t} \mathbf{A}$ and is plotted in Fig. 4. For shortening notations, we put

$$
\mathbf{w}=\mathbf{h}^{t} \mathbf{A}
$$
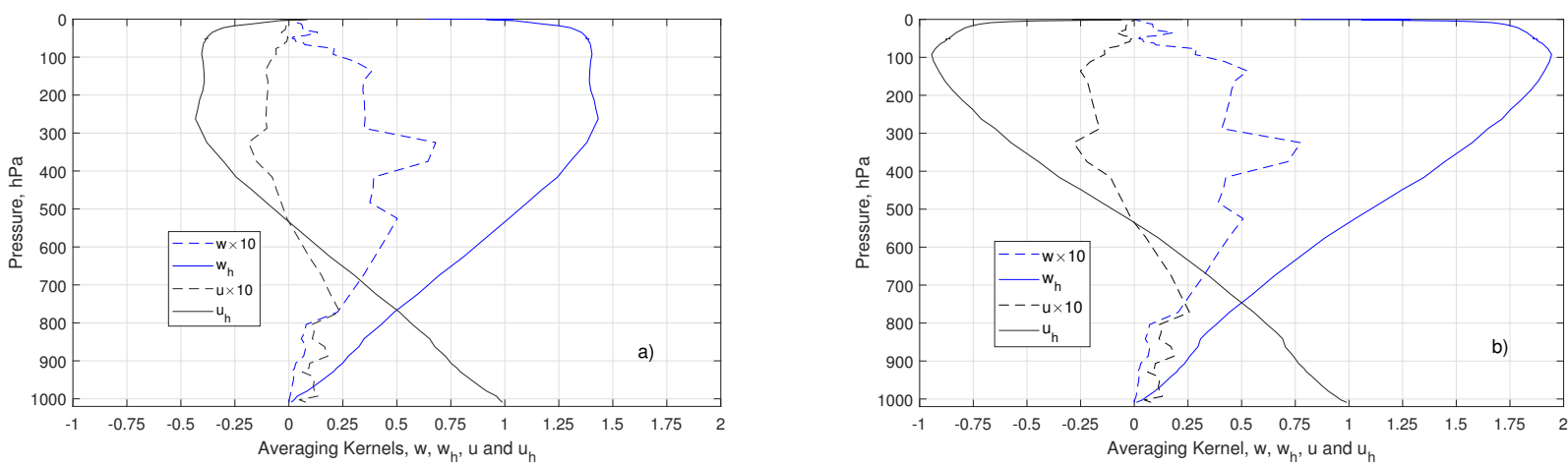

Figure 4. Comparison of the Averaging Kernels, $w, w_{h}, u, u_{h}$ of the OCS integrated column amount. The case shown refers to the GIF (panel a) and Hawaii (panel b) for February 2014. The results shown in figure have been averaged based on 304 (a) and 519 (b) retrievals. Therefore, the values shown are representative of the average condition in February time.

For a perfect retrieval, $\mathbf{A}$ would be the identity matrix, and we should have $\mathbf{h}^{t} \mathbf{A}=\mathbf{w}=\mathbf{h}^{t}$. It is interesting to see that the weights $\mathbf{h}^{t} \mathbf{A}$ remains positive (e.g., see Fig. 4) and different from zero throughout the atmosphere. They are smaller in the lower troposphere because, for each pressure layer i, they are proportional to $\Delta p_{i}$, as shown in Eq. (3), and $\Delta p_{i}$ is not constant along the vertical spatial coordinate because the pressure-altitude grid step is uneven. To avoid this effect, we can normalize the elements of the vector, $\mathbf{h}^{t} \mathbf{A}$ to the corresponding $\Delta p_{i}$ (e.g., see Ref. 22), to have the Averaging Kernel per unit $(1 \mathrm{hPa}$ ) layer pressure depth. Considering the definition of the averaging operator $\mathbf{h}$ (see Eq. (3), the normalization can be achieved simply by defining a new vector $\mathbf{w}_{h}$, whose elements, $w_{h, i}$ are defined according to

$$
w_{h, i}=\frac{w_{i}}{h_{i}}
$$

It should be stressed that in case of a perfect retrieval, we should have $w_{h, i}$, for each layer. For an actual retrieval, $w_{h, i}$ can be greater or lower than 1 , and it is expected to be very small or close to zero in the pressure range from where no contribution is coming to the retrieval. The normalized averaging kernel $\mathbf{w}_{h}$ is also shown in Fig. 4, to be compared with $\mathbf{h}$, and we see that the retrieval $\hat{X}$ receives information from the whole atmosphere, but the lower part where $\mathbf{w}_{h}$ tends linearly to zero. To have further insights into understanding the meaning of $w$ and $w_{h}$ let us consider the linear approximation of the retrieval $\hat{\mathbf{q}}$, namely (e.g., see Ref. 1)

$$
\hat{\mathbf{q}}=\mathbf{q}_{a}+\mathbf{A}\left(\mathbf{q}-\mathbf{q}_{a}\right)=(\mathbf{I}-\mathbf{A}) \mathbf{q}_{a}+\mathbf{A q}
$$

where $\hat{\mathbf{q}}, \mathbf{q}_{a}, \mathbf{q}$, are the estimated background and true OCS profiles, respectively; furthermore, $\mathbf{I}$ is the identity matrix. We can pass to the estimation of the column amount, $\hat{X}$ multiplying both sides by the averaging operator, $\mathbf{h}^{t}$,

$$
\hat{X}=\mathbf{h}^{t} \hat{\mathbf{q}}=\mathbf{h}^{t}(\mathbf{I}-\mathbf{A}) \mathbf{q}_{a}+\mathbf{h}^{t} \mathbf{A q}=X_{b g}+X_{t}
$$

with $X_{b g}$ and $X_{t}$, the contribution for the background and the true profile, respectively. It is interesting to note the background term, $X_{b g}$ can be further split into two terms,

$$
X_{b g}=\mathbf{h}^{t} \mathbf{I} \mathbf{q}_{a}-\mathbf{h}^{t} \mathbf{A} \mathbf{q}_{a}=X_{b g 1}+X_{b g 2}
$$


It is seen that $X_{b g 1}$ depends solely on the background state vector, hence if $\mathbf{q}_{a}$ is a static feature, the term $X_{b g 1}$ cannot change with time and space. The second term, $X_{b g 2}$ depends on the background covariance matrix (again a static feature) and the Jacobian derivative of the atmospheric state. The Jacobian matrix can change with time and space, hence can modulate the overall background term, $X_{b g}$ in a way that alters the time-space dynamic of the signal.

From Eq. (9), we can soon derive the sensitivity of $\hat{X}$ to the true and background profiles,

$$
\frac{\partial \hat{X}}{\partial \mathbf{q}}=\mathbf{h}^{t} \mathbf{A}=\mathbf{w}^{t} ; \quad \frac{\partial \hat{X}}{\partial \mathbf{q}_{a}}=\mathbf{h}^{t}-\mathbf{h}^{t} \mathbf{A}=\mathbf{u}^{t}
$$

It is interesting to note that we have

$$
\mathbf{w}+\mathbf{u}=\mathbf{h}
$$

and

$$
\sum_{i=1}^{N_{L}} w_{i}+\sum_{i=1}^{N_{L}} u_{i}=\sum_{i=1}^{N_{L}} h_{i}
$$

Therefore, if according to the usual meaning of degrees of freedom, e.g. ref. 1, we define

$$
d o f_{\hat{X}}=\sum_{i=1}^{N_{L}} w_{i}
$$

we have that for a given retrieval, a fraction contribution equal to $d o f_{\hat{X}}$ is given by the true profile, whereas a fraction $1-\operatorname{dof}_{\hat{X}}$ is because of the background. Similarly for $\mathbf{w}$, $\mathbf{u}$ can also be normalized to $\mathbf{h}$ yielding the vector $\mathbf{u}_{h}$. With this in mind, Fig. 4a, ehich refers to the GIF area, shows the vectors, $\mathbf{w}, \mathbf{w}_{h}, \mathbf{u}, \mathbf{u}_{h}$. For the case shown, we have $d o f_{\hat{X}}=0.93$, and we note that the background weights, $\mathbf{u}$ changes sign along the vertical so that it sums to +0.07 . Conversely, the weights $\mathbf{w}$ are always positive, and they sum to 0.93 , so that $\mathbf{w}+\mathbf{u}$ is exactly equal to 1. For completeness, Fig. ??b) shows an example for the Hawaii target area (tropics). For this case, we have $d o f_{\hat{X}}=1.02$ and now the background weights, $\mathbf{u}$ sums to -0.02 and those corresponding to $\mathbf{w} 1.02$, so that, again, $\mathbf{w}+\mathbf{u}$ is exactly equal to 1 . The example also shows that $d o f_{\hat{X}}$ can attain values greater than one, but only because the background contribution is negative, in such a way that the constraint imposed by Eq. (12) is satisfied. From a comparison of Fig. 44a and Fig. 4b, we see that the shape of $\mathbf{w}_{h}$ changes according to the latitude belt. In addition, they show that the contribution to the column amount $d o f_{\hat{X}}$ from the true profile comes from everywhere in the atmosphere, although with different weights. The weights tend to be greater in the upper-middle troposphere and goes to zero at the surface.

The set of equations from 9 to 14 is the most important methodological result of the present study. They show that the background contribution $X_{b} g$ has a direct dependence on the averaging kernels, $\mathbf{A}$, which in turn depend on the atmospheric state. This aspect was also evidenced in Ref. 23 when dealing with the column amount retrieval of $\mathrm{CO}_{2}$, and it cannot be simply ignored, as it will be exemplified in the results section.

\section{RESULTS}

\subsection{1 Assessing the d.o.f. dynamic-variability on the time-behavior of retrievals}

Figure 5 shows the comparison of the IASI OCS (monthly means) and the in situ GIF observations (monthly averaged). Although the range and cycle amplitude are correct, we see a delay between the two-time series, which is of the order of 2 months. IASI data points exhibit a peak in February and a trough in August, whereas the same characteristics are shown in April and October, respectively, for the GIF observations. If we consider the linear correlation between the two series, we find it equal to 0.45 .

What we see in Fig. 5 is a subtle aspect of the seasonal variability of the information content, that is $d o f_{\hat{X}}$. In effect, especially for land surface retrievals outside the tropical belt, the strong seasonal contrast of the surface temperature and emissivity can modulate Jacobian, hence the kernel w, and introduce a seasonal cycle, which overlaps that of the OCS. This seasonal effect is exemplified in Fig. 6, where we show the monthly time series of 


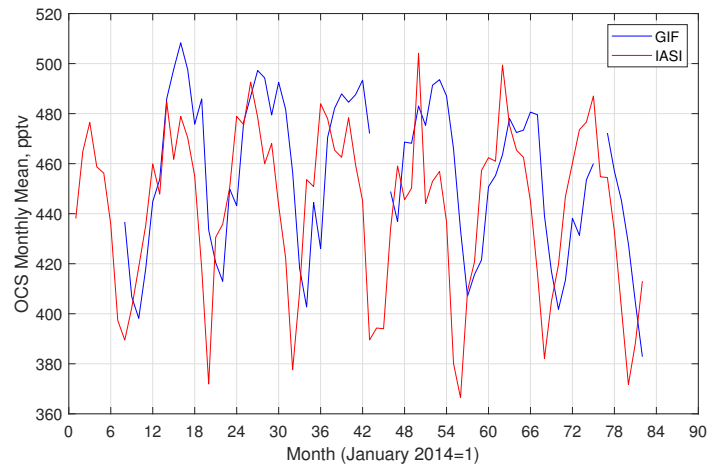

Figure 5. Comparison of IASI and in situ OCS (monthly means) for the GIF target area. White segments on the blue line (GIF in situ) correspond to missing data.

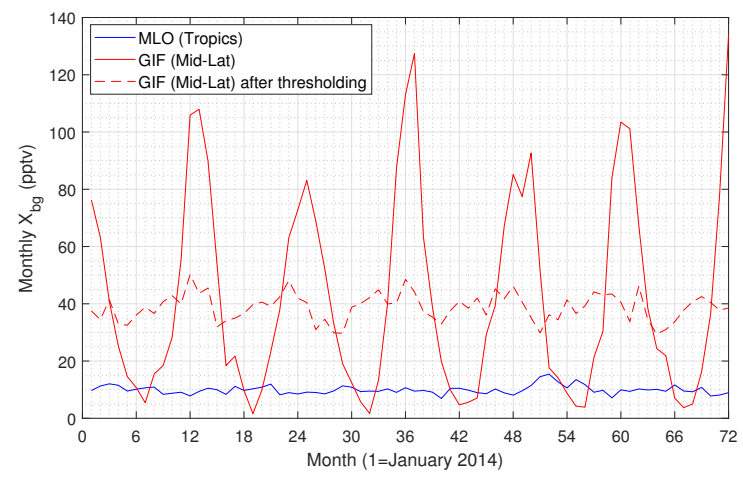

Figure 6. Background contribution to the column amount $X_{b g}$ as a function of the month (2014 to 2019 ) for the MLO (tropics) and a GIF (Mid-Lat stations), respectively.

$X_{b g}$ for the period 2014-2019 for the GIF station. For comparison, the same figure also shows the case of MLO (tropical belt).

In effect, Fig. 6 shows that the phenomenon is most seen on land and outside the tropical belt, in this latter case, the uniform climate all around the year does not introduce seasonal variability in $\mathbf{A}$, hence $d o f_{\hat{X}}$ remains fairly constant. We stress again that the background state is a static feature, not changing with time; therefore, if we see seasonal effects in $X_{b g}$, it is because they are introduced by a seasonal behavior of $d o f_{\hat{X}}$. It also has to be stressed that the seasonal cycle shown by $X_{b g}$ has peaks in February and troughs in August; therefore, the OCS dynamics (see Fig. 5) is dominated by the $X_{b g}$ cycle rather than the data. We see that for the tropical station, the contribution from the background remains confined to the values of $1-2 \%$ of $\hat{X}$. Conversely, for the mid-lat station, $X_{b g}$ can reach values as large as $20-25 \%$ and is strongly modulated with the seasons. In effect, this behavior is the result of the variations in $d_{0} f_{\hat{X}}$ with the season. As further exemplified in Fig. $7, d o f_{\hat{X}}$ undergoes very little changes at tropics, whereas at mid-lat it can reach values as low as 0.80 .

To avoid the inclusion of spurious seasonal variations in the time series of $\hat{X}$ we need to perform the analysis at constant $d o f_{\hat{X}}$. Towards this objective, the retrievals have been filtered according to the criterion

$$
0.98 \leq d o f_{\hat{X}} \leq 1.02
$$

The effect of this criterion once applied to the GIF station is seen again in Fig. 6 and Fig. 7 Again, we see that the background contribution goes down to $\approx 8 \%$, with no critical cyclic components.

Figure 8 is the same as Fig. 5, but now the OCS retrievals have been filtered according to the condition of Eq. (15). In addition, the IASI OCS time series have been smoothed with a moving average window with a time 


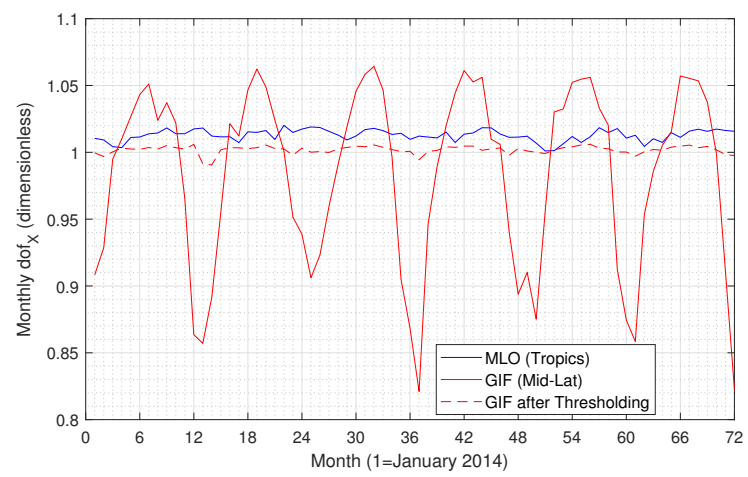

Figure 7. Monthly series of $\operatorname{dof}_{\hat{X}}(2014$ to 2019) for a tropical and a mid-lat station, respectively.

span of 3 months to reduce its random variability. We see that the cycle amplitude seen from IASI is smaller than that explained from the in situ observations. However, now the cycle goes into phase, and the correlation is raised to 0.74 .

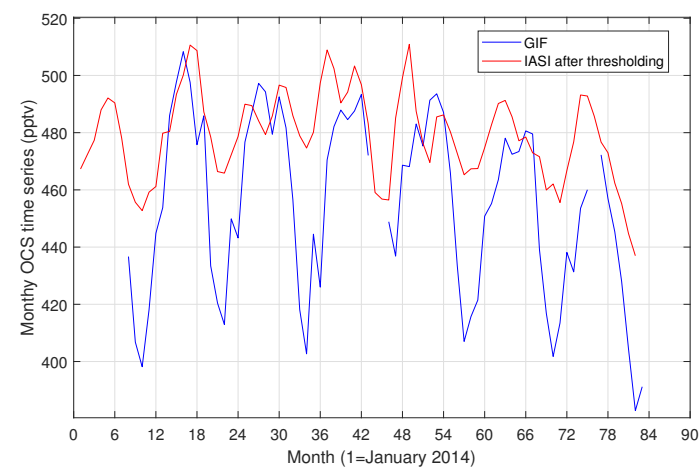

Figure 8. Comparison of IASI and in situ OCS (monthly means) for the GIF target area. IASI data have been filtered according to the criterion of Eq. (15). White segments on the blue line (in situ data) correspond to missing data.

IASI retrievals show an amplitude of the cycle, smaller than in situ GIF data because the IASI estimate is integrated over the whole atmospheric column, whereas GIF measurements are taken in the boundary layer. Also, the atmospheric column above the GIF area can receive the contribution from the many local sources in North-East Europe because of air mass transport. The vertical distribution is neither uniform nor stationary. In contrast, when we analyze a target area far from anthropogenic sources, the vertical profile shows a smaller gradient. Therefore, we expect a more consistent satellite agreement with in situ observations. When we look at the results of Fig. 9, which refers to the MLO tropical station, we see that IASI and in situ agree with each other much better than for the GIF case; the cycle is better reproduced, and the correlation reaches the value of 0.82. We also stress that this time, the criterion of Eq. (15) has not been applied.

\subsection{Assessing the d.o.f. spatial-variability on the space-behavior of retrievals}

Figure 10 shows a scatter map of $d o f_{\hat{X}}$ for the Euro-Med target area (see, e.g., Fig. 2). The mapping does not involve any averaging operation; therefore, it shows the $d o f_{\hat{X}}$ corresponding to each IASI sounding. It should be noted that at given locations many IASI soundings can overlap, which have different $d o f_{\hat{X}}$.

From Fig. 10a (no thresholding applied), we see that $d o f_{\hat{X}}$ has a large variability over land, where it can be as low as 0.4. The more considerable variability is seen in the East part of Europe, but also over mountains. In contrast, over the ocean, $d o f_{\hat{X}}$ is uniformly close to 1 . Figure $10 \mathrm{~b}$ shows the scatter map of $d o f_{\hat{X}}$ after 


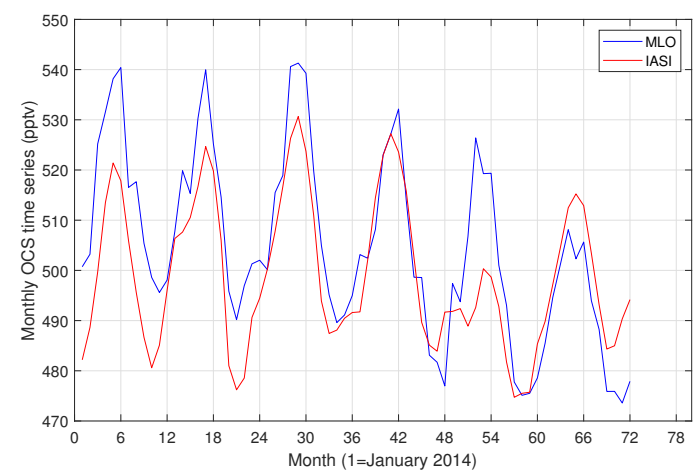

Figure 9. Comparison of IASI and in situ OCS (monthly means) for the MLO target area.
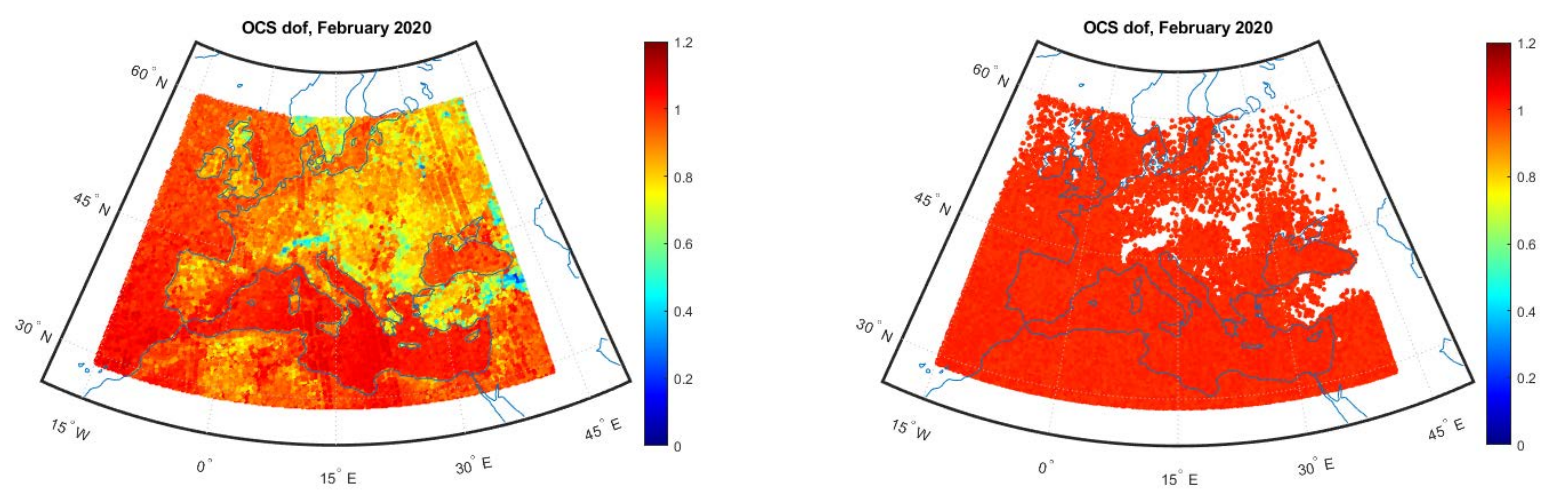

Figure 10. Scatter map of $d o f_{\hat{X}}$ without (left) and with thresholding (right) according to the criterion of Eq. (15).

thresholding with the criterion of Eq. (15). It is seen now that valid IASI soundings Eq. $d o f_{\hat{X}} \approx 1$ do not cover a large part of Eastern Europe on the map. If we try to build up a map without thresholding, the result would be highly biased, with patterns reproducing the d.o.f. variability, rather than the natural spatial dynamics of OCS. In fact, according to Eq. (9), the smaller the $d o f_{\hat{X}}$, the larger the bias.

We also stress that Fig. 10a might lead to the wrong conclusion that we should have significant gaps over Spain and some parts of North Africa because of the many $d o f_{\hat{X}}$ below thresholds. However, as said before, IASI footprints can overlap; therefore, after thresholding, the remaining good footprints are revealed.

An analysis in terms of the background contribution, $X_{b g}$ is provided in Fig. 11. The scatter maps show the ratio (see Eq. (9)

$$
100 \times \frac{X_{b g}}{\hat{X}}
$$

for both cases without (left) and with (right) thresholding according to Eq. (15). It is seen that without thresholding, the background state can dominate the final estimate with a fractional contribution larger than 50\%. In addition, the presence of thick patterns over mountains and the North-East side of Europe are identified. In contrast, when we apply thresholding, the contribution of $X_{b g}$ to the final estimate is less than 10\%. Again, we see that thresholding leaves gaps primarily at mountains sites and over the Eastern part of Europe.

Finally, Fig. 12 shows the OCS monthly map after thresholding to avoid artifacts added by the background rather that actual observations. The map has been obtained by using the methodology of optimal interpolation developed in [21, 22], with two different length scales, which determine the spatial correlation extend, namely 

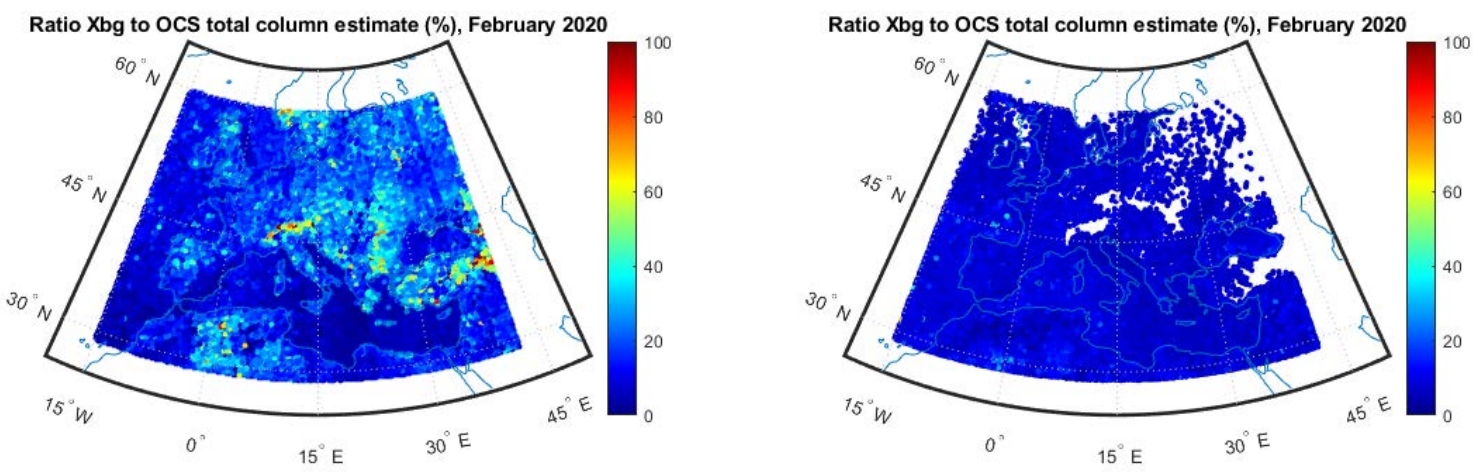

Figure 11. Scatter map of the ratio defined by Eq. (16) without (left) and with thresholding (right) according to the criterion of Eq. (15).

$1^{\circ}$ and $0.1^{\circ}$. With the $1^{\circ}$ map we can fill the gaps left after thresholding (see Eq. (15). However, the spatial resolution is degraded at the expense of the uniform coverage. In contrast, the $0.1^{\circ}$ map still contains gaps, but now the spatial resolution is improved of a factor of ten.
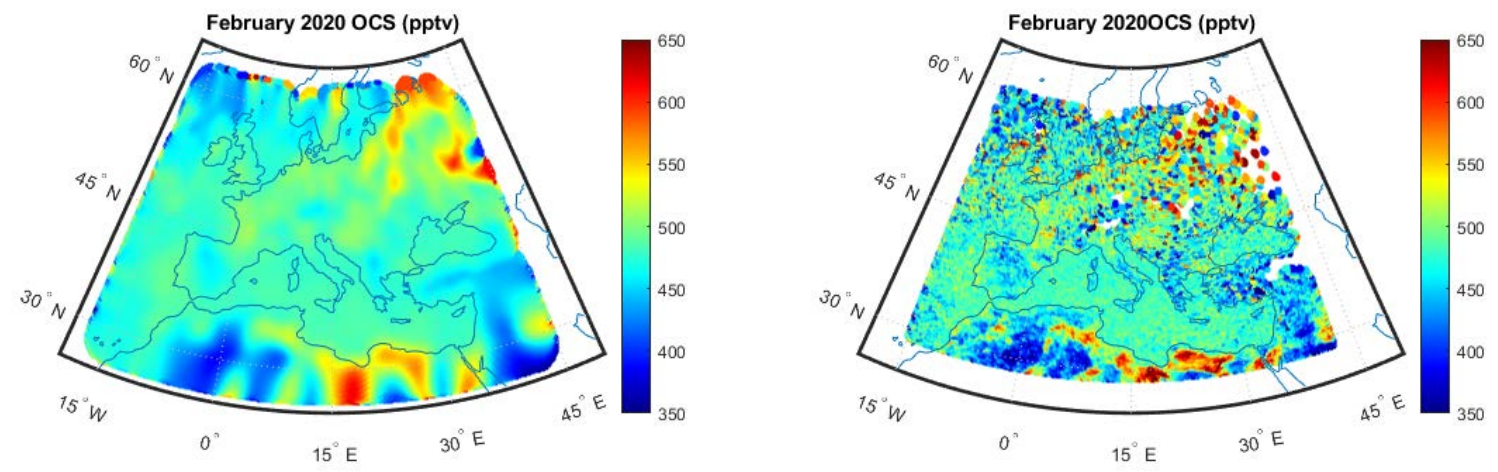

Figure 12. Monthly OCS map at different spatial resolution and after thresholding application; (left) the correlation length scale is $1^{\circ}$; (right) the correlation length scale is $0.1 \circ$.

Despite the different spatial resolution, we see that there is a relatively large concentration of OCS over the North-East corner of Europe, which could be the results of emissions from biomass and coal power stations plants. We also see a striking feature over the North-Africa, the delta of the Nile, and the Sinai region. The OCS pattern over North Africa has also been identified by concurrent analysis with IASI (see, e.g., Ref. 8) and other satellite instrumentation (e.g., Ref. 24). It is not yet clear if the pattern is locally produced, e.g., because of the presence of oil and gas fields, or it is a result of atmospheric transport, as suggested in Ref. 24. However, our analysis now shows that it is not an artifact of d.o.f. variability but is a real phenomenon still to be understood as an additional, until now unknown, source of OCS. In effect, the much more resolved $0.1^{\circ}$. The OCS pattern over North Africa has also been identified by concurrent analysis with IASI (see, e.g., Ref. 8) and other satellite instrumentation (e.g., Ref. 24). It is not yet clear if the pattern is locally produced, e.g., because of the presence of oil and gas fields, or it is a result of atmospheric transport, as suggested in Ref. 24. However, our analysis now shows that it is not an artifact of d.o.f. variability but is a real phenomenon still to be understood as 
an additional, until now unknown, source of OCS. In effect, the much more resolved $0.1^{\circ}$ map shows that the pattern follows the regions where oil and gas methane fields are active. It is well known that the oil and gas refinery produces $\mathrm{CS}_{2}$, which upon oxidation with $\mathrm{OH}$, transforms to OCS (see Ref. 25,26). Also, the potential $\mathrm{CS}_{2}$ source from the Libyan oil and gas field seems to have been overlooked until now as a potential source of OCS (e.g., see Ref. 27). In effect, the most recent OCS inventory ${ }^{27}$ of anthropogenic OCS fluxes identifies the delta of the Nile river (as seen in our analysis) as a source of OCS but fails to show a possible contribution from the Libyan oil fields.

\section{CONCLUSIONS}

We have analyzed and assessed the effect of d.o.f. variability over retrievals from satellite high infrared spectral resolution sensors. Although the methodology has been exemplified with the case of atmospheric OCS, the approach is quite general and can be extended to any atmospheric parameter retrieved, e.g., on the basis of OE. We have shown that even in the case the background constraint is a static feature of the inverse system, it can introduce dynamical biases, which can alter the natural annual cycle of the given parameters. The problem also affects spatial fields because the atmospheric state can rapidly change because of a complex surface and meteorological conditions, especially over land. The issue should not be confused with the "smoothing" introduced by the constraint, which depends solely on the covariance matrix of the background (a static feature itself). The effect we are dealing with has to do with the variability of the state vector. Also, in our analysis, the covariance matrix is prescribed to have large variances to implement a very loose constraint.

We have shown that coherent $d o f_{\hat{X}}$ patterns can extend to large spatial scales, introducing biases that cannot be smoothed by considering reliable neighbour data points. An adequate way to come up with this bias is to apply a threshold criterion, which selects soundings with $d o f_{\hat{X}} \approx 1$ and ensure that retrievals are mainly depending on the data alone.

Our analysis of the OCS map on February 2020 shows a relevant atmospheric concentration of OCS over North-East Europe, which is in agreement with the most recent inventory of anthropogenic OCS sources. However, we have also found a relatively large and thick concentration of OCS over the Libyan oil and gas fields, which seems to be previously unnoticed. Finally, it is fair to say that this thick concentration could also result from atmospheric transport; therefore, more analysis is needed to draw a firm conclusion.

\section{ACKNOWLEDGMENTS}

In situ observations recorded at Mauna Loa Observatory in Hawaii have been downloaded from the web page http://www.esrl.noaa.gov/gmd/obop/mlo/. The station is part of the National Oceanic and Atmospheric Administration (NOAA), the Earth System Research Laboratory (ESRL) and the Global Monitoring Division (GMD). We give credit to the National Oceanic and Atmospheric Administration (NOAA)/Global Monitoring Division (GMD) in Boulder as a source of the data. IASI has been developed and built under the responsibility of the Centre National d'Etudes Spatiales (CNES, France). It is flown onboard the Metop satellites as part of the EUMETSAT Polar System. The IASI L1C data are received through the EUMETCast near real-time data distribution service. This research was carried out in the framework of the project 'OT4CLIMA' which was funded by the Italian Ministry of Education, University and Research (D.D. 2261 del 6.9.2018, PON R\&I 2014-2020 and FSC).

\section{REFERENCES}

[1] Rodgers, C. D., "Inverse methods for atmopsheric sounding: Theory and practice," World Scientific, Singapore, 2000 (2000).

[2] Hilton, F., Armante, R., August, T., Barnet, C., Bouchard, A., Camy-Peyret, C., Capelle, V., Clarisse, L., Clerbaux, C., Coheur, P.-F., Collard, A., Crevoisier, C., Dufour, G., Edwards, D., Faijan, F., Fourrié, N., Gambacorta, A., Goldberg, M., Guidard, V., Hurtmans, D., Illingworth, S., Jacquinet-Husson, N., Kerzenmacher, T., Klaes, D., Lavanant, L., Masiello, G., Matricardi, M., McNally, A., Newman, S., Pavelin, E., Payan, S., Péquignot, E., Peyridieu, S., Phulpin, T., Remedios, J., Schlüssel, P., Serio, C., Strow, L., Stubenrauch, C., Taylor, J., Tobin, D., Wolf, W., and Zhou, D., "Hyperspectral earth observation from IASI: Five years of accomplishments," Bulletin of the American Meteorological Society 93(3), 347-370 (2012). 
[3] Asaf, D., Rotenberg, E., Tatarinov, F., Dicken, U., Montzka, S., and Yakir, D., "Ecosystem photosynthesis inferred from measurements of carbonyl sulphide flux," Nature Geoscience 6(3), 186-190 (2013).

[4] Berry, J., Wolf, A., Campbell, J. E., Baker, I., Blake, N., Blake, D., Denning, A. S., Kawa, S. R., Montzka, S. A., Seibt, U., Stimler, K., Yakir, D., and Zhu, Z., "A coupled model of the global cycles of carbonyl sulfide and co2: A possible new window on the carbon cycle," Journal of Geophysical Research: Biogeosciences 118(2), 842-852 (2013).

[5] Campbell, J. E., Whelan, M. E., Seibt, U., Smith, S. J., Berry, J. A., and Hilton, T. W., "Atmospheric carbonyl sulfide sources from anthropogenic activity: Implications for carbon cycle constraints," Geophysical Research Letters 42(8), 3004-3010 (2015).

[6] Kuai, L., Worden, J., Kulawik, S. S., Montzka, S. A., and Liu, J., "Characterization of aura tes carbonyl sulfide retrievals over ocean," Atmospheric Measurement Techniques 7(1), 163-172 (2014).

[7] Kuai, L., Worden, J. R., Campbell, J. E., Kulawik, S. S., Li, K.-F., Lee, M., Weidner, R. J., Montzka, S. A., Moore, F. L., Berry, J. A., Baker, I., Denning, A. S., Bian, H., Bowman, K. W., Liu, J., and Yung, Y. L., "Estimate of carbonyl sulfide tropical oceanic surface fluxes using aura tropospheric emission spectrometer observations," Journal of Geophysical Research: Atmospheres 120(20), 11,012-11,023 (2015).

[8] Vincent, R. A. and Dudhia, A., "Fast retrievals of tropospheric carbonyl sulfide with iasi," Atmospheric Chemistry and Physics 17(4), 2981-3000 (2017).

[9] Liuzzi, G., Masiello, G., Serio, C., Venafra, S., and Camy-Peyret, C., "Physical inversion of the full IASI spectra: Assessment of atmospheric parameters retrievals, consistency of spectroscopy and forward modelling," Journal of Quantitative Spectroscopy and Radiative Transfer 182, 128 - 157 (2016).

[10] Camy-Peyret, C., Liuzzi, G., Masiello, G., Serio, C., Venafra, S., and Montzka, S., "Assessment of IASI capability for retrieving carbonyl sulphide (OCS)," Journal of Quantitative Spectroscopy and Radiative Transfer 201, 197 - 208 (2017).

[11] Serio, C., Masiello, G., Mastro, P., Cersosimo, A., Pasquariello, P., and Venafra, S., "Simultaneous retrieval of OCS, and CO2 from the IASI shortwave spectral band: assessment of the accuracy of the retrieval products and validation with in situ observations.," in [Remote Sensing of Clouds and the Atmosphere XXV], Comerón, A., Kassianov, E. I., Schäfer, K., Picard, R. H., Weber, K., and Singh, U. N., eds., 11531, $1-13$, International Society for Optics and Photonics, SPIE (2020).

[12] Montzka, S. A., Calvert, P., Hall, B. D., Elkins, J. W., Conway, T. J., Tans, P. P., and Sweeney, C., "On the global distribution, seasonality, and budget of atmospheric carbonyl sulfide (cos) and some similarities to co2," Journal of Geophysical Research: Atmospheres 112(D9) (2007).

[13] Belviso, S., Reiter, I. M., Loubet, B., Gros, V., Lathière, J., Montagne, D., Delmotte, M., Ramonet, M., Kalogridis, C., Lebegue, B., Bonnaire, N., Kazan, V., Gauquelin, T., Fernandez, C., and Genty, B., "A topdown approach of surface carbonyl sulfide exchange by a mediterranean oak forest ecosystem in southern france," Atmospheric Chemistry and Physics 16(23), 14909-14923 (2016).

[14] Belviso, S., Rmonet, M., Kazan, V., Pison, I., Berchet, A., and et al., "A top-down approach of sources and non-photosynthetic sinks of carbonyl sulfide from atmospheric measurements over multiple years in the paris region (france)," PLoS ONE 15(2) (2020).

[15] Masiello, G., Serio, C., and Shimoda, H., "Qualifying img tropical spectra for clear sky," Journal of Quantitative Spectroscopy and Radiative Transfer 77(2), 131 - 148 (2003).

[16] Amato, U., Lavanant, L., Liuzzi, G., Masiello, G., Serio, C., Stuhlmann, R., and Tjemkes, S. A., "Cloud mask via cumulative discriminant analysis applied to satellite infrared observations: scientific basis and initial evaluation," Atmospheric Measurement Techniques 7(10), 3355-3372 (2014).

[17] Mastro, P., Pasquariello, P., Masiello, G., and Serio, C., "Cloud detection from IASI hyperspectral data: a statistical approach based on neural networks," in [Remote Sensing of Clouds and the Atmosphere XXV], Comerón, A., Kassianov, E. I., Schäfer, K., Picard, R. H., Weber, K., and Singh, U. N., eds., 11531, 40 49, International Society for Optics and Photonics, SPIE (2020).

[18] Amato, U., Masiello, G., Serio, C., and Viggiano, M., "The $\sigma$-IASI code for the calculation of infrared atmospheric radiance and its derivatives," Environmental Modelling \& Software 17(7), 651-667 (2002).

[19] Shephard, M. W., Clough, S. A., Payne, V. H., Smith, W. L., Kireev, S., and Cady-Pereira, K. E., "Performance of the line-by-line radiative transfer model (LBLRTM) for temperature and species retrievals: IASI case studies from JAIVEx," Atmospheric Chemistry and Physics 9, 7397-7417 (2009). 
[20] Alvarado, M. J., Payne, V. H., Mlawer, E. J., Uymin, G., Shephard, M. W., Cady-Pereira, K. E., Delamere, J. S., and Moncet, J.-L., "Performance of the line-by-line radiative transfer model (lblrtm) for temperature, water vapor, and trace gas retrievals: recent updates evaluated with IASI case studies," Atmospheric Chemistry and Physics 13(14), 6687-6711 (2013).

[21] Carissimo, A., De Feis, I., and Serio, C., "The physical retrieval methodology for IASI: the $\delta$-IASI code," Environmental Modelling \& Software 20(9), 1111-1126 (2005).

[22] Connor, B. J., Boesch, H., Toon, G., Sen, B., Miller, C., and Crisp, D., "Orbiting carbon observatory: Inverse method and prospective error analysis," Journal of Geophysical Research: Atmospheres 113(D5) (2008).

[23] Serio, C., Masiello, G., Camy-Peyret, C., and Liuzzi, G., "CO2 spectroscopy and forward/inverse radiative transfer modelling in the thermal band using IASI spectra," Journal of Quantitative Spectroscopy and Radiative Transfer 222-223, 65 - 83 (2019).

[24] Glatthor, N., Höpfner, M., Baker, I. T., Berry, J., Campbell, J. E., Kawa, S. R., Krysztofiak, G., Leyser, A., Sinnhuber, B.-M., Stiller, G. P., Stinecipher, J., and von Clarmann, T., "Tropical sources and sinks of carbonyl sulfide observed from space," Geophysical Research Letters 42(22), 10,082-10,090 (2015).

[25] Rich, A. L. and Patel, J. T., "Carbon disulfide (CS2) mechanisms in formation of atmospheric carbon dioxide (CO2) formation from unconventional shale gas extraction and processing operations and global climate change," Environmental Health Insights 9s1, EHI.S15667 (2015). PMID: 25987843.

[26] Khan, A., Razis, B., Gillespie, S., Percival, C., and Shallcross, D., "Global analysis of carbon disulfide (CS2) using the 3-d chemistry transport model stochem[j]," AIMS Environmental Science 4, 484 (2017).

[27] Zumkehr, A., Hilton, T. W., Whelan, M., Smith, S., Kuai, L., Worden, J., and Campbell, J. E., "Global gridded anthropogenic emissions inventory of carbonyl sulfide," Atmospheric Environment 183, 11-19 (2018). 